\title{
Artifact Reduction in Compressed Images Based on Region Homogeneity Constraints Using the Projection Onto Convex Sets Algorithm
}

\author{
Chaminda Weerasinghe, Alan Wee-Chung Liew, and Hong Yan, Senior Member, IEEE
}

\begin{abstract}
In this paper, a novel projection onto convex sets (POCS) method is presented for the suppression of blocking and ringing artifacts in a compressed image that contains homogeneous regions. A new family of convex smoothness constraint sets is introduced, using the uniformity property of image regions. This set of constraints allows different degrees of smoothing in different regions of the image, while preserving the image edges. The regions are segmented using the fuzzy c-means algorithm, which allows ambiguous pixels to be left unclassified. Experimental results on JPEG compressed images demonstrate that the proposed algorithm yields visually superior images compared to several of the recently reported POCS deblocking algorithms for the class of images considered.
\end{abstract}

Index Terms-Blocking artifacts, fuzzy C-means, image deblocking, projection onto convex sets, region homogeneity constraints.

\section{INTRODUCTION}

$\mathbf{T}$ HE BLOCK discrete cosine transform (BDCT) is a commonly used transform for both still and moving image coding, such as in JPEG and MPEG. It is based on dividing an image into small blocks, normally $8 \times 8$, computing the DCT of each block, and quantizing the DCT coefficients according to a predefined quantization table. The DCT has become a prominent technique for image compression since it is an asymptotic approximation to the optimal Karhunen-Loeve Transform (KLT) when the statistical properties of the image can be represented by a first-order Markov model. However, a major drawback of BDCT-based techniques is that the compressed image exhibit visually annoying blocking and ringing artifacts, especially at high compression ratio. Blocking artifacts appear due to quantizing low-frequency coefficients, which gives rise to discontinuity between intensities of two adjacent blocks, whereas ringing artifacts are caused by discarding high-frequency coefficients.

Projection onto convex sets (POCS) is a method for solving many constraint satisfaction and optimization problems and has

Manuscript received January 15, 2002; revised July 16, 2002. This work was supported in part by the Hong Kong Research Grant Council (Project CityU 1088/00E). This paper was recommended by Associate Editor D. W. Lin.

C. Weerasinghe was with the School of Electrical and Information Engineering, University of Sydney, NSW 2006, Australia. He is now with Motorola Australian Research Center, Botany, NSW 2019, Australia.

A. W.-C. Liew is with the Department of Computer Engineering and Information Technology, City University of Hong Kong, Kowloon Tong, Hong Kong (e-mail: itwcliew@cityu.edu.hk).

H. Yan is with the School of Electrical and Information Engineering, University of Sydney, NSW 2006, Australia and also with Department of Computer Engineering and Information Technology, City University of Hong Kong, Kowloon Tong, Hong Kong.

Digital Object Identifier 10.1109/TCSVT.2002.804881 many applications in signal and image processing problems [1]-[8]. It has also been intensively investigated in the past for coding artifacts suppression. The method works by iteratively updating the BDCT data, using a priori knowledge about the compressed image. Two prominent constraints have been used, namely the quantization constraint in the DCT domain, and the smoothness constraint in the spatial domain. In the formulation proposed by Zakhor [9], it is argued that the frequency components, which are higher than a given cutoff frequency, are mainly due to the blocking artifacts. However, the original image may contain high-frequency components above the given cutoff frequency; hence, the method may produce an excessively smoothed image. This method is equivalent to blind low-pass filtering regulated only by the quantization constraint. In the formulation proposed by Yang et al. [10], smoothing is only performed at the block boundaries. However, the blocking artifact is mainly caused by the differences between intensities of two adjacent blocks, rather than only the pixels in the block boundaries. Therefore, this technique results in insufficient suppression of coding artifacts. In the method proposed by Paek et al. [11], it is assumed that the original image is highly correlated, where the global frequency characteristics in two adjacent blocks are very similar to the local frequency characteristics of each block for smooth region. However, this assumption does not apply to blocks containing edges, thus preventing the suppression of blocking artifacts for those blocks.

Blocking and ringing artifact suppression is usually approached based on either block boundary pixels or adjacent blocks. The smoothness constraint can be applied to the homogeneous regions. This region smoothness constraint, which is applied on image regions, gives a more global smoothing then smoothness constraint for blocks or block boundary pixels. Edges will not be oversmoothed, since they form boundaries of regions and are not affected by the region smoothness constraint. None of the previous methods have explored the possibility of incorporating homogeneity of segmented regions of the image to regulate the smoothing on the compressed image. Therefore, by incorporating the region smoothness constraint, better suppression of blocking and ringing artifacts can be achieved for the class of image considered.

\section{CONSTRAined IMAge ReCOVERY}

In this section, the recovery of the original image from the compressed data is attempted using the theory of POCS. The imposed constraints include the transmitted BDCT coefficients 
with the prior knowledge on the quantizer and the available $a$ priori knowledge in the spatial domain, in the form of a region smoothness constraint.

\section{A. Theory of POCS}

The conceptual basis of POCS is that the available constraints can be represented as convex sets in a Hilbert space, $H$ [1], [11]. For image reconstruction, the Hilbert space is the set of all two-dimensional square integrable functions with the usual inner product

$$
(f, g)=\iint f(x, y) g^{*}(x, y) d x d y
$$

where $g^{*}$ represents the complex conjugate. In image reconstruction problems, every known property of the original $f$ restricts it to lie in a corresponding closed convex subset of $H$. In general, therefore, $m$ such properties will generate $m$ well-defined closed convex sets $C_{i}$, where $i=1,2, \ldots, m$, and therefore

$$
f \in C_{0}=\bigcap_{i=1}^{m} C_{i} .
$$

The intersection $C_{0}$ is also closed convex and contains $f$. Consequently, irrespective of whether $C_{0}$ contains elements other than $f$, the problem of reconstructing $f$ from its $m$ properties is equivalent to that of finding at least one point belonging to $C_{0}$.

If the projection operator $P_{i}$, projecting onto its respective convex set $C_{i}$ is effectively realizable for $i=1,2, \ldots, m$, the problem is recursively solvable. The rule that assigns to every $x \in H$ its nearest neighbor $f \in C_{i}$ defines the nonlinear projection operator $P_{i}: H \rightarrow C_{i}$ unambiguously, i.e.,

$$
\left\|x-P_{i} x\right\|=\min _{f \in C_{i}}\|x-f\| .
$$

Consider the composition operator

$$
T=P_{m} P_{m-1} \cdots P_{1} .
$$

Then the following recursion

$$
x_{n}=T^{n} x
$$

converges to an element in $C_{0}$ as $n=0 \rightarrow \infty$, for any arbitrary starting point $x \notin C_{0}$. For details on POCS theory, readers are directed to [1], [12].

\section{B. Convex Sets and Projectors}

POCS-based image recovery algorithms require two sources of information to be represented as convex sets: 1) the constraint sets with the information derived from the available data and 2) the constraint sets with the prior knowledge supplied to complement the available data. In the case of JPEG compressed images, the data constraint in the BDCT domain for an $N \times N$ block can be formulated as follows [9]:

$$
C_{D}=\left\{f: \hat{F}_{i}^{\min } \leq(D f)_{i} \leq \hat{F}_{i}^{\max }, \quad \forall i=1,2, \ldots, N^{2}\right\}
$$

where $\hat{F}$ are the quantized BDCT coefficients of $N^{2} \times 1$ image vector $f$. $D$ is an $N^{2} \times N^{2}$ unitary BDCT transformation matrix. $\hat{F}_{i}^{\min }$ and $\hat{F}_{i}^{\max }$ are the $i$ th minimum and maximum allowable BDCT coefficients as determined by the quantizer's $i$ th quantization interval $\Delta_{i}$ and the $i$ th quantized BDCT coefficient $\hat{F}_{i}$, i.e.,

$$
\begin{aligned}
& \hat{F}_{i}^{\min }=\hat{F}_{i} \Delta_{i}-0.5 \Delta_{i} \\
& \hat{F}_{i}^{\max }=\hat{F}_{i} \Delta_{i}+0.5 \Delta_{i} .
\end{aligned}
$$

The projection of $f$ onto the set $C_{D}$ is given by

$$
P_{D} f=D^{T} D f=D^{T} F
$$

where

$$
F_{i}= \begin{cases}\hat{F}_{i}^{\min }, & \text { if }(D f)_{i}<\hat{F}_{i}^{\min } \\ \hat{F}_{i}^{\max }, & \text { if }(D f)_{i}>\hat{F}_{i}^{\max } \\ (D f)_{i}, & \text { otherwise. }\end{cases}
$$

The smoothness constraint in the spatial domain, based on the region homogeneity within the image, is formulated as follows:

$$
C_{s}=\left\{f:\left\|r_{f}-M_{r_{f}}\right\| \leq \varepsilon\right\}
$$

where $r_{f}$ is a homogeneous region in the image $f$ (i.e., $r_{f} \subset f$ ), and $M_{r_{f}}$ is given by

$$
M_{r_{f}}=\frac{1}{k} \sum_{i=0}^{k-1} r_{f}(i)
$$

with $k$ representing the number of elements in $r_{f}$ and $\varepsilon$ the bounding value.

1) Convexity of $C_{s}$ : A set $C$ is convex if and only if it contains every convex combinations of its elements, i.e., for every $f \in C$ and $g \in C,[\alpha f+(1-\alpha) g] \in C$ for all $0 \leq \alpha \leq 1$. Let $f_{1}, f_{2} \in C_{s}$ and let $f_{3}=\alpha f_{1}+(1-\alpha) f_{2}$ where $\alpha \in[0,1]$. Therefore, $r_{f_{3}}=\alpha r_{f_{1}}+(1-\alpha) r_{f_{2}}$. According to (12), it can be derived that $M_{r_{f_{1}}}=\alpha M_{r_{f_{2}}}+(1-\alpha) M_{r_{f_{3}}}$. Then by the triangle inequality and the linearity of the Euclidean norm, we obtain

$$
\begin{aligned}
& \left\|r_{f_{3}}-M_{r_{f_{3}}}\right\| \\
& \quad=\left\|\alpha r_{f_{1}}+(1-\alpha) r_{f_{2}}-\alpha M_{r_{f_{1}}}-(1-\alpha) M_{r_{f_{2}}}\right\| \\
& \quad=\left\|\alpha\left(r_{f_{1}}-M_{r_{f_{1}}}\right)+(1-\alpha)\left(r_{f_{2}}-M_{r_{f_{2}}}\right)\right\| \\
& \quad \leq \alpha\left\|r_{f_{1}}-M_{r_{f_{1}}}\right\|+(1-\alpha)\left\|r_{f_{2}}-M_{r_{f_{2}}}\right\| \\
& \quad \leq \alpha \varepsilon+(1-\alpha) \varepsilon=\varepsilon .
\end{aligned}
$$

Therefore, $f_{3} \in C_{s}$ and hence the set $C_{s}$ is convex.

2) Closedness of $C_{s}$ : A set is closed if and only if it contains all its limit points. Let $\left\{f_{n}\right\}$ be a sequence in $C_{s}$ such that $\lim _{n \rightarrow \infty} f_{n} \rightarrow f^{*}$, which implies that $r_{f_{n}} \rightarrow r_{f^{*}}$. According to (12), it can be shown that $M_{r_{f^{*}}}=M_{r_{f^{*}}-r_{f_{n}}}+M_{r_{f_{n}}}$. Therefore

$$
\begin{aligned}
& \left\|r_{f^{*}}-M_{r_{f^{*}}}\right\| \\
& \quad=\left\|\left(r_{f^{*}}-r_{f_{n}}\right)+r_{f_{n}}-M_{r_{f^{*}}-r_{f_{n}}}-M_{r_{f_{n}}}\right\| \\
& \quad \leq\left\|\left(r_{f^{*}}-r_{f_{n}}\right)-M_{r_{f^{*}}-r_{f_{n}}}\right\|+\left\|r_{f_{n}}-M_{r_{f_{n}}}\right\| .
\end{aligned}
$$


When $n \rightarrow \infty$

$$
\lim _{n \rightarrow \infty}\left\|r_{f^{*}}-M_{r_{f^{*}}}\right\| \leq\left\|r_{f_{n}}-M_{r_{f_{n}}}\right\| \leq \varepsilon
$$

Thus, $f^{*} \in C_{s}$ and the set $C_{s}$ is closed.

3) Projection of $C_{s}$ : Let $g \notin C_{s}$ and $f \in C_{s}$, where $f$ is the projection of $g$ onto $C_{s}$, i.e., $f=P_{s} g$, where $P_{s}$ is the projection operator. Let the regions $r_{g} \subset g$ and $r_{f} \subset f$. Therefore, $\left\|r_{g}-M_{r_{g}}\right\|>\varepsilon$ and $\left\|r_{f}-M_{r_{f}}\right\|=\varepsilon$. Consider the Lagrange functional $J\left(r_{f}\right)$

$$
J\left(r_{f}\right)=\left\|r_{g}-r_{f}\right\|^{2}+\lambda\left[\left\|r_{f}-M_{r_{f}}\right\|^{2}-\varepsilon^{2}\right]
$$

where $\lambda$ is the Lagrange multiplier. Using (12) and taking the partial derivative of $J\left(r_{f}\right)$ with respect to a particular $r_{f}(i)$ and setting it to zero, we obtain

$$
\frac{\partial J\left(r_{f}\right)}{\partial r_{f}(i)}=2\left(r_{f}(i)-r_{g}(i)\right)+2 \lambda\left(r_{f}(i)-M_{r_{f}}\right)\left(1-\frac{1}{k}\right)=0
$$

which yields

$$
r_{f}(i)=\frac{r_{g}(i)+\lambda(1-1 / k) M_{r_{f}}}{1+\lambda(1-1 / k)}
$$

From (12) and (18), $M_{r_{f}}=M_{r_{g}}$. Therefore, (18) can be rewritten as

$$
r_{f}(i)=\frac{r_{g}(i)+\lambda(1-1 / k) M_{r_{g}}}{1+\lambda(1-1 / k)} .
$$

To find $\lambda$, we invoke the condition

$$
\left\|r_{f}-M_{r_{f}}\right\|=\varepsilon
$$

which yields

$$
\lambda=\frac{k}{k-1}\left[\frac{\left\|r_{g}-M_{r_{g}}\right\|}{\varepsilon}-1\right]
$$

Using (19) and (21), we obtain

$$
r_{f}(i)=\frac{\varepsilon}{\left\|r_{g}-M_{r_{g}}\right\|} r_{g}(i)+\left[1-\frac{\varepsilon}{\left\|r_{g}-M_{r_{g}}\right\|}\right] M_{r_{g}} .
$$

Therefore, the projection of $g$ onto the set $C_{s}$ can be realized by

$$
r_{f}(i)= \begin{cases}\alpha r_{g}(i)+(1-\alpha) M_{r_{g}}, & \text { if }\left\|r_{g}-M_{r_{g}}\right\|>\varepsilon \\ r_{g}(i), & \text { otherwise }\end{cases}
$$

where $\alpha=\varepsilon /\left\|r_{g}-M_{r_{g}}\right\|$. If there are $n$ number of homogeneous regions contained within the image, this information can be formulated as $n$ different convex sets $C_{S_{1}}, C_{S_{2}}, \ldots, C_{S_{n}}$ with bounding values $\varepsilon_{1},, \varepsilon_{2}, \ldots, \varepsilon_{n}$, which enables different degrees of smoothing in different regions.

\section{EXTRACTION OF HOMOGENEOUS REGIONS}

In order to implement the new region smoothness constraint introduced in this paper, the homogeneous regions need to be extracted from the compressed image. This task is performed using the fuzzy c-means (FCM) clustering algorithm for labeling similar colored regions. Fuzzy techniques for image segmentation have been chosen due to the following reasons.

- POCS solution can be adversely affected by mis-labeling, which can occur with hard c-means (HCM) clustering or thresholding techniques.

- It is more appropriate to label ambiguous pixels, i.e., those having near-uniform membership values in all clusters, as unclassified, rather than assigning them to one of the clusters which could significantly affect the mean vector of that cluster.

Pixels that are labeled as ambiguous are automatically estimated during the POCS iterations, without enforcing the region smoothness constraint on them. The extraction of homogeneous regions consists of three stages, namely initial cluster centers estimation, fuzzy c-means clustering, and pixels labeling.

\section{A. Initial Cluster Centers Estimation}

The initial cluster centers estimation is based on the histograms of the hue component of the color image. To obtain the hue component $H$, the following transformation from RGB space is used [18]:

$$
H=\cos ^{-1} \frac{0.5((R-G)+(R-B))}{\left((R-G)^{2}+(R-B)(G-B)\right)^{0.5}} .
$$

If the denominator is zero, then $H$ is set to $\pi / 2$. If $B>G$, $H=2 \pi-H$. Finally, $H$ is converted to degree by multiplying with $180 / \pi$. The hue histogram is obtained by quantizing the hue angle in degree into 180 bins. To smooth out small or closely spaced peaks, a [0.25 0.50 .25$]$ smoothing mask is run through the histogram. The initial cluster centers are then obtained from the smoothed hue histogram by locating the significant peaks in the histogram and adopting the RGB values that correspond to those peaks. A peak at a particular bin is declared as significant if it is separated from other significant peaks by at least three bins and has more than 100 elements in the bin. For closely located peaks (i.e., $<3$ bins), the one with more elements is selected over the others.

\section{B. FCM Clustering}

The FCM clustering algorithm has been widely used in many pattern recognition problems [13]-[16]. The objective of FCM algorithm is to cluster the feature vectors $X$ by finding the local minimum of the generalized within cluster sum of squared error objective function

$$
J_{m}(U, V)=\sum_{k=1}^{n} \sum_{i=1}^{c} u_{i k}^{m}\left|x_{k}-v_{i}\right|^{2}
$$

subject to the constraint $\sum_{i=1}^{c} u_{i k}=1, \forall k$. In this application, $X=\left\{x_{1}, x_{2}, \ldots, x_{n}\right\}$ is the finite set of color information, where $x_{k} \in R^{3}$ is a three-dimensional RGB color 
TABLE I

THREE QUANTIZATION TABLES FOR BDCT COMPRESSION

Q1

$\begin{array}{llllllll}50 & 60 & 70 & 70 & 90 & 120 & 255 & 255\end{array}$

$\begin{array}{lllllllll}60 & 60 & 70 & 96 & 130 & 255 & 255 & 255\end{array}$

$70 \quad 70 \quad 80 \quad 120200255255255$

$70 \quad 96 \quad 120145255255255255$

$90 \quad 130200255255255255255$

120255255255255255255255

255255255255255255255255

255255255255255255255255
Q2

$\begin{array}{lllllll}86 & 59 & 54 & 86 & 129216255255\end{array}$ $\begin{array}{llllll}64 & 64 & 75 & 102140255255255\end{array}$

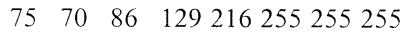

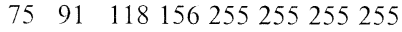
$97 \quad 118199255255255255255$ 129189255255255255255255 255255255255255255255255 255255255255255255255255
Q3

110130150192255255255255 130150192255255255255255 150192255255255255255255 192255255255255255255255 255255255255255255255255 255255255255255255255255 255255255255255255255255 255255255255255255255255

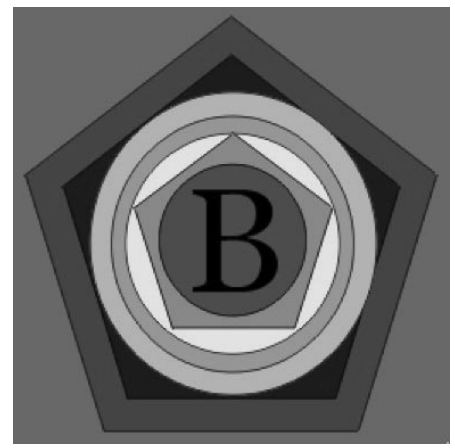

(a)

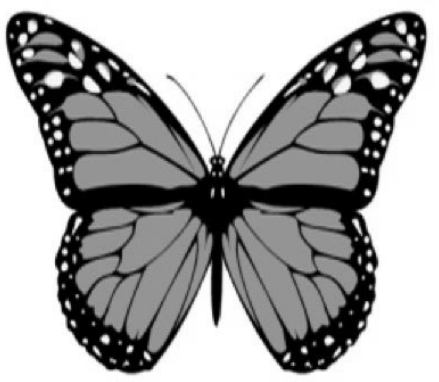

(b)

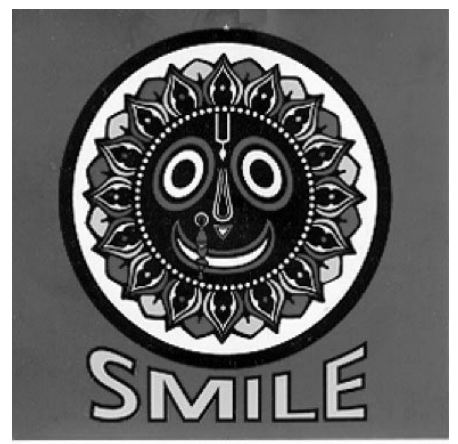

(c)

Fig. 1. Original test images of (a) "logo," (b) "butterfly," and (c) "smile."

\section{EXPERIMENTAL RESULTS}

The proposed image recovery algorithm has been tested on three different color images, i.e., "logo," "butterfly," and "smile," of size $256 \times 256$ shown in Fig. 1. The images are BDCT encoded using the three quantization tables Q1, Q2, Q3 listed in Table I. The blocking and ringing artifacts are clearly visible in the compressed image (using quantization table Q2) 


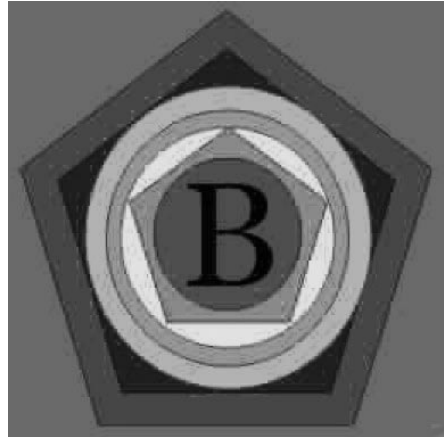

(a)

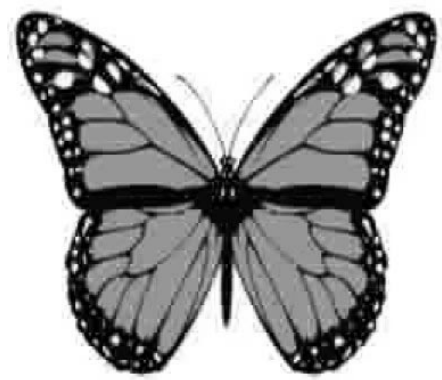

(b)

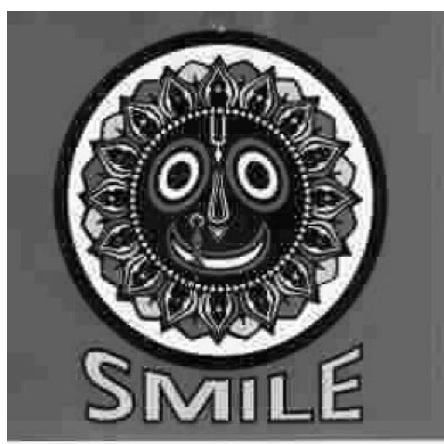

(c)

Fig. 2. BDCT compressed test images using the quantization table Q2.

shown in Fig. 2. The performance of the proposed method is compared to that of the three POCS-based methods, namely the method of Zakhor [9], Yang et al. [10], and Paek et al. [11]. The smoothness bound in our algorithm is set to $\varepsilon=0.5 \sqrt{3 k}$, where $k$ is the number of elements in each region.

Fig. 3 shows the deblocked images that correspond to the images in Fig. 2 produced by the proposed algorithm. The blocking and ringing artifacts can be observed to have been largely suppressed. Table II gives the quantitative results of the comparative study in term of peak signal-to-noise ratio (PSNR) in decibels. For the three test images, the proposed algorithm has better performance over the algorithms of Paek and Zakhor. For the "smile" and "butterfly" image, Yang's algorithm is slightly better in PSNR than the proposed algorithm, whereas for the "logo" image, the proposed algorithm is better than Yang's algorithm. The slight decrease in performance of our algorithm for the "smile" and "butterfly" image is due to the existence of fine homogeneous regions (and the associated region boundaries) in the original images. These fine regions

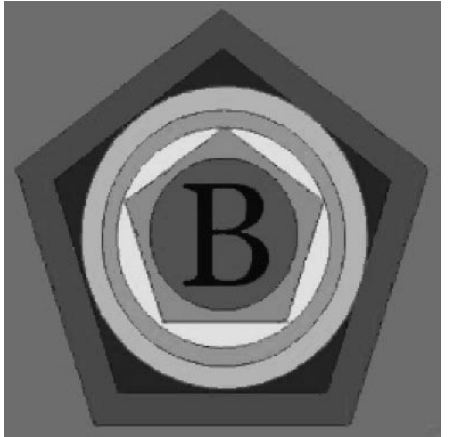

(a)

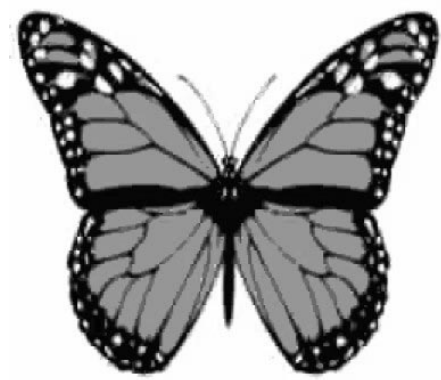

(b)

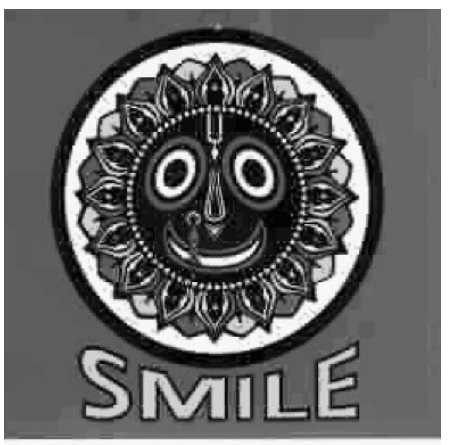

(c)

Fig. 3. Deblocked images that correspond to the compressed images in Fig. 2.

(and edges) are smeared out and destroyed after BDCT and lossy quantization, making them difficult to be labeled and segmented correctly into homogeneous regions by the FCM algorithm. Most of the pixels in these regions are thus classified as ambiguous and the region smoothness constraint is not applied to them. A more sophisticated region classification and segmentation procedure should be able to alleviate this difficulty.

To compare the subjective quality of the recovered images, part of the test images are shown in Figs. 4 and 5. It can be clearly seen that the proposed method is more effective in eliminating both the blocking and ringing artifacts compared to the other three methods, while preserving the edge details. In Fig. 6, the PSNR improvement is compared among the four methods for increasing number of iterations for the image "logo." The method of Zakhor actually produced results that have lower PSNR than the compressed image. As noted by [17], Zakhor's method of low-pass filtering does not actually constitute a projection operation. The low-pass filtering actu- 
TABLE II

PSNR PERFORMANCE (IN DECIBELS) OF OUR ALgORITHM IN COMPARISON With THREE OTHER POCS-BASED DEBLOCKING ALGORITHMS IN THE LitERATURE

\begin{tabular}{c|c|c|c|c|c|c|c|c|c}
\hline & \multicolumn{3}{|c|}{$\log$ s } & \multicolumn{3}{c|}{ butterfly } & \multicolumn{3}{c}{ smile } \\
\hline & Q1 & Q2 & Q3 & Q1 & Q2 & Q3 & Q1 & Q2 & Q3 \\
\hline BDCT Compressed & 24.19 & 23.54 & 21.75 & 24.87 & 24.34 & 21.14 & 21.27 & 20.87 & 18.28 \\
\hline Paek[11] & 24.42 & 23.68 & 21.92 & 25.07 & 24.50 & 21.28 & 21.35 & 20.94 & 18.36 \\
\hline Yang[10] & 24.46 & 23.71 & 22.08 & 25.50 & 24.85 & 21.72 & 21.55 & 21.11 & 18.60 \\
\hline Zakhor[9] & 23.29 & 22.68 & 21.23 & 23.45 & 22.96 & 20.21 & 19.97 & 19.59 & 17.30 \\
\hline Proposed & 25.25 & 24.57 & 22.53 & 25.31 & 24.83 & 21.31 & 21.53 & 21.10 & 18.46 \\
\hline
\end{tabular}

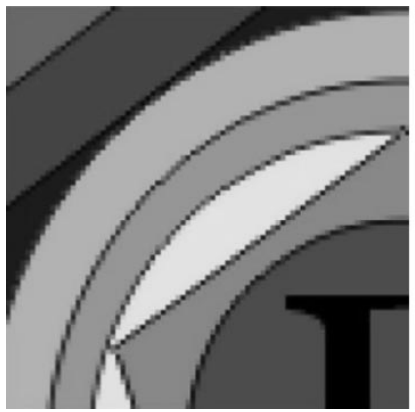

(a)

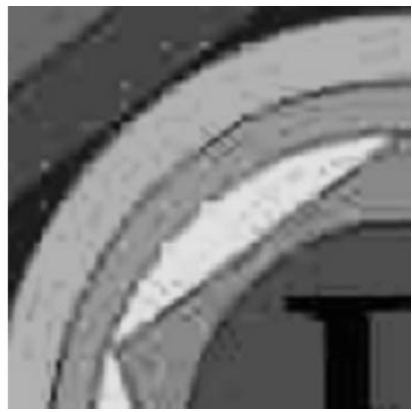

(b)

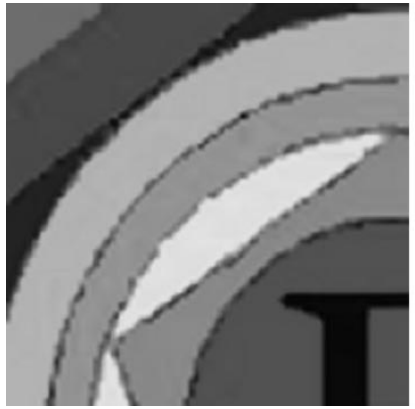

(c)

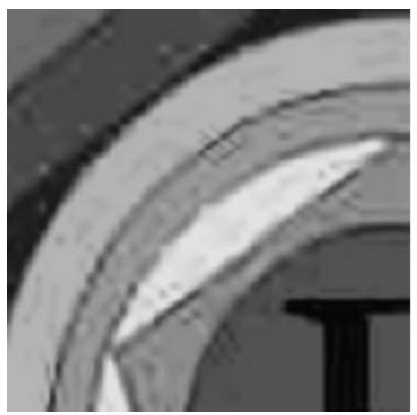

(e)

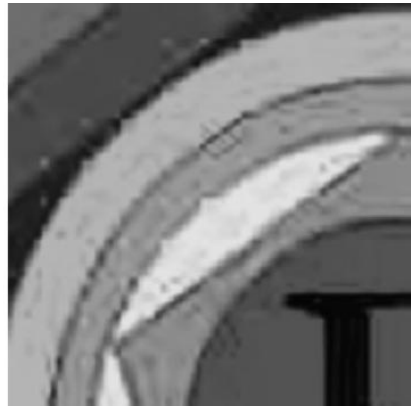

(d)

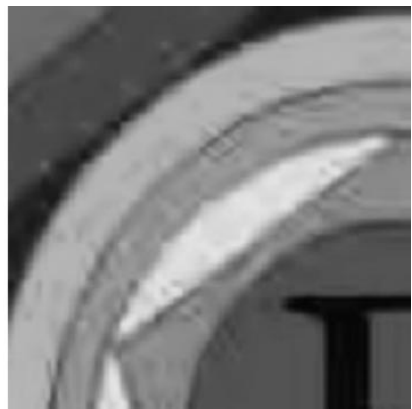

(f)

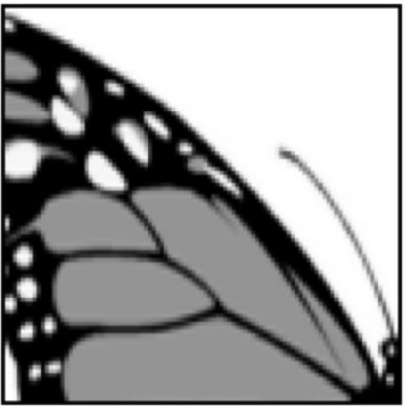

(a)

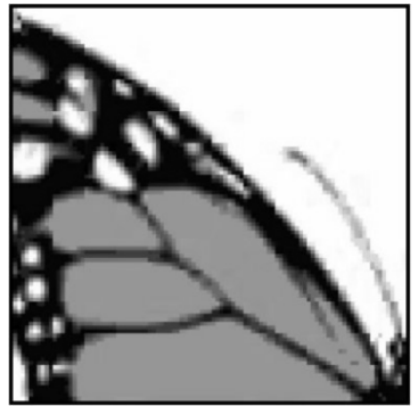

(c)

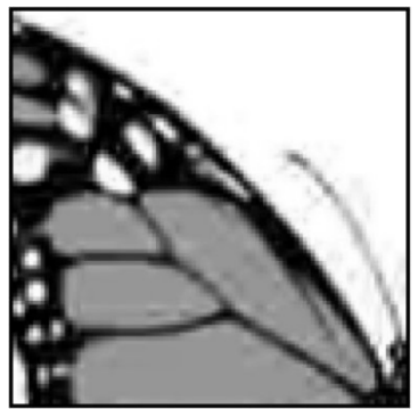

(e)

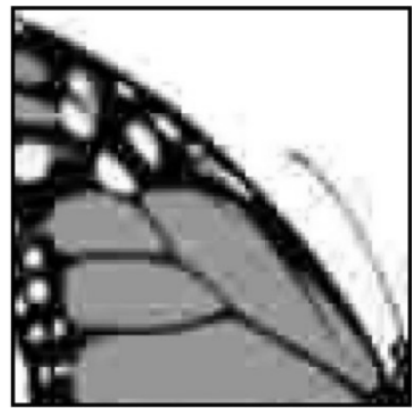

(b)

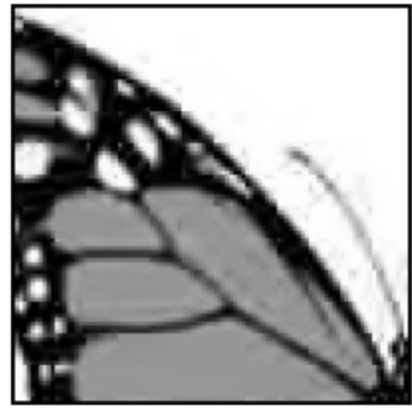

(d)

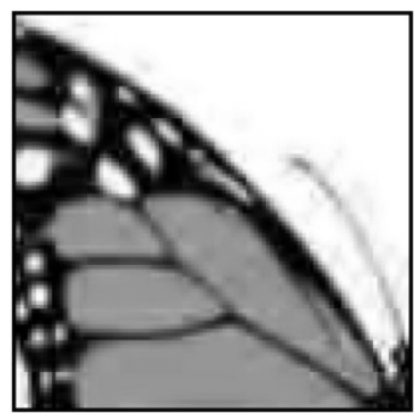

(f)

Fig. 4. Part of deblocked "logo" image, showing the visual quality of the reconstructions. Left to right, top to bottom: (a) original image; (b) BDCT compressed image with Q2; (c) deblocked image using our method; (d) deblocked image using Paek's method; (e) deblocked image using Yang's method; and (f) deblocked image using Zakhor's method.

ally blurs the edges, giving rise to the inferior results as shown in our experiment.

The proposed algorithm is conceptually very simple. A major part of the algorithm is the segmentation of the homogeneous regions, which is done by using the FCM algorithm. Although the FCM can be quite costly in terms of computation, the finding of good initial cluster centers allows the clustering algorithm to converge rapidly. Good enough clustering results can typically be obtained within five iterations. The actual projection onto

Fig. 5. Part of deblocked "butterfly" image, showing the visual quality of the reconstructions. Left to right, top to bottom: (a) original image; (b) BDCT compressed image with Q2; (c) deblocked image using our method; (d) deblocked image using Paek's method; (e) deblocked image using Yang's method; and (f) deblocked image using Zakhor's method.

the region smoothness constraint set is computationally simpler than Paek's smoothness projection (which requires repeated one-dimensional DCT computations) and Zakhor's low-pass filtering operation. However, the significant improvement in the visual quality of the final image for the class of images considered justifies our approach.

Our method has been specially designed to exploit the homogeneous region property in the image. The idea of smooth region constraint has to be extended to deal with image having 


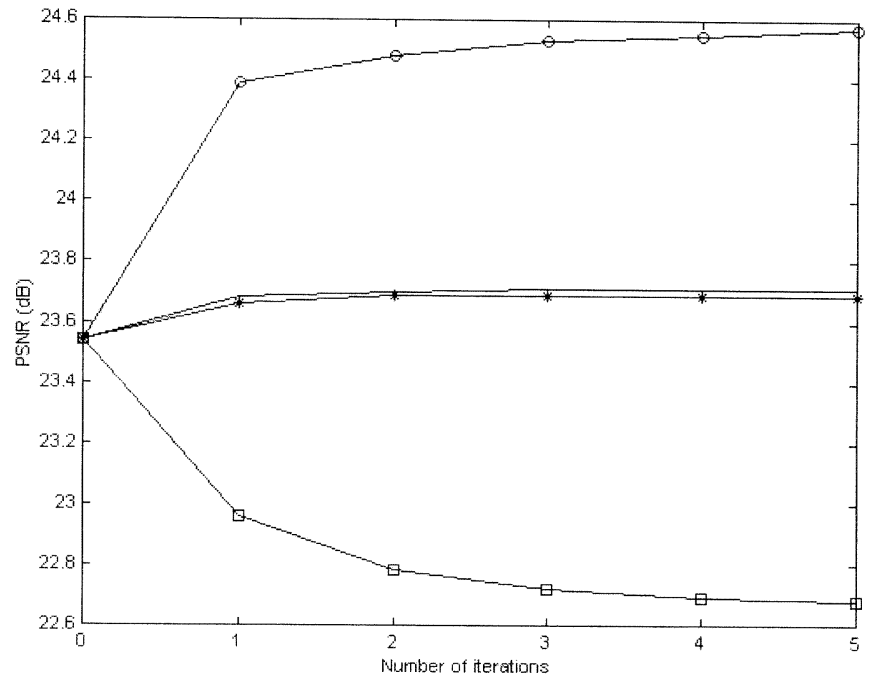

Fig. 6. PSNR versus iterations for the four POCS techniques for BDCT compressed "logo" image using Q2. Starting from the highest PSNR at the fifth iteration: our method, Yang's method, Paek's method, and Zakhor's method.

nonuniform regions. One possible way to extend the method is to define smoothly varying regions instead of uniform regions. This is currently being investigated. Finally, although we have described the POCS-based method to deal with image artifacts produced by the BDCT, the method is generally applicable to other transform coding, such as wavelet transforms.

\section{CONCLUSIONS}

A POCS-based deblocking algorithm is proposed for the class of color images having uniform regions. The algorithm exploits the special property of this class of image, namely, the regions of uniform color value, to formulate region smoothness constraint set for the image recovery problem. Since correct segmentation of the uniform regions is essential to the successful application of the proposed algorithm, a segmentation procedure using the fuzzy c-means algorithm is presented. The fuzzy segmentation allows pixels with low membership values to be left unclassified, thus minimizing the risk of assigning them to the wrong region. Simulation results show that both the blocking and ringing artifacts can be significantly reduced using the proposed algorithm, while preserving the edges of the image. The proposed algorithm performs better than the POCS-based methods of Zakhor [9] and Paek [11] while comparable or better than Yang's [10] method, both in terms of objective and subjective quality, for the class of images considered.

\section{REFERENCES}

[1] H. Stark and Y. Yang, Vector Space Projections, A Numerical Approach to Signal and Image Processing, Neural Nets, and Optics. New York: Wiley, 1998.

[2] M. Hedley, H. Yan, and D. Rosenfeld, "Motion artifact correction in MRI using generalized projections," IEEE Trans. Medical Imaging, vol. 10, pp. 40-46, 1991.

[3] M. Hedley and H. Yan, "Suppression of slice selection axis motion artifacts in MRI," IEEE Trans. Medical Imaging, vol. 11, pp. 233-237, 1992.

[4] Y. Yang and H. Stark, "Solutions of several color-matching problems using projection theory," J. Opt. Soc. Amer. A, vol. 11, pp. 1-8, 1994.
[5] Y. Yang, N. P. Galatsanos, and H. Stark, "A project-based approach to the blind deconvolution problem," J. Opt. Soc. Amer. A, vol. 11, pp. 89-96, 1994.

[6] C. Weerasinghe and H. Yan, "Correction of motion artifact caused by motion of constant angular velocity," Signal Processing, vol. 70, pp. 103-114, 1998.

[7] _ "An improved algorithm for rotational motion artifact suppression in MRI," IEEE Trans. Medical Imaging, vol. 17, pp. 310-317, 1998.

[8] A. W. C. Liew and N. F. Law, "Reconstruction from 2-D wavelet transform modulus maxima using projection," Proc. Inst. Elect. Eng.-Vision, Image and Signal Processing, vol. 147, no. 2, pp. 176-184, 2000.

[9] A. Zakhor, "Iterative procedures for reduction of blocking effects in transform image coding," IEEE Trans. Circuits Syst. Video Technol., vol. 2, pp. 91-95, Feb. 1992.

[10] Y. Yang, N. P. Galatsanos, and A. K. Katsaggelos, "Regularized reconstruction to reduce blocking artifacts of block discrete cosine transform compressed images," IEEE Trans. Circuits Syst. Video Technol., vol. 3 , pp. 421-432, 1993.

[11] H. Paek, R. C. Kim, and S. U. Lee, "On the POCS-based postprocessing technique to reduce the blocking artifacts in transform coded images," IEEE Trans. Circuits Syst. Video Technol., vol. 8, pp. 358-367, June 1998.

[12] D. C. Youla and H. Webb, "Image restoration by the method of convex projections: Part 1-Theory," IEEE Trans. Medical Imaging, vol. MI-1, pp. 81-94, 1982.

[13] J. C. Bezdek, Pattern Recognition With Fuzzy Objective Function Algorithms. New York: Plenum Press, 1981

[14] Z. Chi, H. Yan, and T. D. Pham, Fuzzy Algorithms, With Applications to Image Processing and Pattern Recognition. Singapore: World Scientific, 1996.

[15] Y. W. Lim and S. U. Lee, "On the color image segmentation algorithm based on the thresholding and the fuzzy c-means techniques," Pattern Recognit., vol. 23, no. 9, pp. 935-952, 1990.

[16] A. W. C. Liew, S. H. Leung, and W. H. Lau, "Fuzzy image clustering incorporating spatial continuity," Proc. Inst. Elect. Eng._Vision, Image and Signal Processing, vol. 147, no. 2, pp. 185-192, 2000.

[17] S. J. Reeves and S. L. Eddins, "Comments on 'Iterative procedures for reduction of blocking effects in transform image coding'," IEEE Trans. Circuits Syst. Video Technol., vol. 3, pp. 439-440, 1993.

[18] R. C. Gonzalez and R. E. Woods, Digital Image Processing. Reading, MA: Addison-Wesley, 1992.

Chaminda Weerasinghe received the B.E. degree (Honors Class 1 with University Medal) from the University of Wollongong, Wollongong, Australia, and the Ph.D. in image processing from The University of Sydney, NSW, Australia.

$\mathrm{He}$ is currently a Senior Research Engineer with Motorola Australian Research Center. His main research interests are in color image processing for CMOS image sensors, panoramic image stitching, stereoscopic visualization, and image scaling for display devices, as well as imaging algorithm development for specific applications and prototyping in hardware using FPGA devices.

Alan Wee-Chung Liew received the B.E. degree (with first-class honors) in electrical and electronic engineering from the University of Auckland, Auckland, New Zealand, in 1993, and the Ph.D. degree in electronic engineering from the University of Tasmania, Tasmania, Australia, in 1997.

$\mathrm{He}$ is currently a Senior Research Fellow in the Department of Computer Engineering and Information Technology, City University of Hong Kong. His research interests include image processing, pattern recognition, and bioinformatics.

Hong Yan (S'88-M'89-SM'93) received the B.E. degree from Nanking Institute of Posts and Telecommunications, Nanking, China, in 1982, the M.S.E. degree from the University of Michigan, Ann Arbor, in 1984, and the Ph.D. degree from Yale University, New Haven, CT, in 1989, all in electrical engineering.

In 1982 and 1983, he worked on signal detection and estimation as a graduate student and Research Assistant at Tsinghua University, Tsinghua, China. From 1986 to 1989, he was a Research Scientist at General Network Corporation, New Haven, CT, where he worked on design and optimization of computer and telecommunications networks. He joined the University of Sydney, Sydney, NSW, Australia, in 1989 and became Professor of Imaging Science in 1997. $\mathrm{He}$ is currently Professor of Computer Engineering at City University of Hong Kong. His research interests include image processing, pattern recognition and bioinformatics. He is author or co-author of one book and over 200 refereed technical papers in these areas.

Dr. Yan is a Fellow of the International Association for Pattern Recognition (IAPR) and the Institution of Engineers, Australia (IEAust) and a member of the International Society for Computational Biology (ISCB). 